\title{
Linear and nonlinear optical properties of carbon nanotube-coated single-mode optical fiber gratings
}

\author{
Guillermo E. Villanueva, ${ }^{1, *}$ Michael B. Jakubinek, ${ }^{2}$ Benoit Simard, ${ }^{2}$ Claudio J. Oton, ${ }^{1}$ \\ Joaquín Matres, ${ }^{1}$ Li-Yang Shao, ${ }^{3}$ Pere Pérez-Millán, ${ }^{1}$ and Jacques Albert ${ }^{3}$ \\ ${ }^{1}$ Nanophotonics Technology Center, Universidad Politécnica de Valencia, Camino de Vera s/n, 46022 Valencia, Spain \\ ${ }^{2}$ Steacie Institute for Molecular Sciences, National Research Council Canada, 100 Sussex Drive, Ottawa Ontario K1A oR6, Canada \\ ${ }^{3}$ Advanced Photonic Components Group, Carleton University, 1125 Colonel By Drive, Ottawa Ontario K1S 5B6, Canada \\ ${ }^{*}$ Corresponding author: guivilib@ntc.upv.es
}

Received February 18, 2011; accepted April 11, 2011;

posted April 21, 2011 (Doc. ID 142944); published May 31, 2011

\begin{abstract}
Single-wall carbon nanotube deposition on the cladding of optical fibers has been carried out to fabricate an all-fiber nonlinear device. Two different nanotube deposition techniques were studied. The first consisted of repeatedly immersing the optical fiber into a nanotube supension, increasing the thickness of the coating in each step. The second deposition involved wrapping a thin film of nanotubes around the optical fiber. For both cases, interaction of transmitted light through the fiber core with the external coating was assisted by the cladding mode resonances of a tilted fiber Bragg grating. Ultrafast nonlinear effects of the nanotube-coated fiber were measured by means of a pump-probe pulses experiment. @ (c) 2011
\end{abstract}

OCIS codes: $\quad 060.3735,060.4370,160.4236$.

Single-wall carbon nanotubes (SWNTs) have found innovative applications in the optical field due to their nonlinear properties in the near IR. Depending on the chirality of SWNTs, they can be either metallic or semiconducting, the latter being the desired behavior for nonlinear optical performance. Many optical applications could benefit from using SWNTs as nonlinear materials, such as noise suppression, wavelength conversion, and passive mode-locking [1-3] . A challenge when designing SWNT-based devices is incorporating the nanotubes in such a way as to enhance their interaction with light. One common method involves dispersing SWNTs in a solvent and spraying the solvent onto substrates in order to produce a nanotube film in the middle of a light beam [3]. Some inherent drawbacks in these configurations are the need for alignment and focusing stages, low SWNT burn thresholds, and low nonlinear interaction length. Solutions that overcome these challenges are based on SWNT deposition onto optical fibers. Tapered, $D$-shaped, and hollow optical fibers have been proposed to take advantage of the evanescent field interaction with SWNTs, distributing that interaction along the fiber length [4-6]. Nevertheless, manufacturing and handling these kinds of fibers is quite complex and delicate. In this work we propose for the first time SWNT deposition on standard optical fiber cladding for nonlinear applications. Interaction of light propagating through the fiber core with the outer SWNT coating is achieved via cladding mode resonances in a tilted fiber Bragg grating (TFBG). Two nanotube deposition methods have been carried out: dip-coating of the optical fiber in a SWNT suspension and wrapping of a SWNT film around the optical fiber.

A TFBG is a kind of grating in which the index modulation planes are not orthogonal to the fiber axis but form a particular tilt angle. This inclination enhances the coupling of light from core mode to counterpropagating cladding mode resonances. Consequently, the grating transmission response is a multinotch response consist- ing of numerous cladding mode resonances in addition to the core mode resonance [see Fig. 1(a)]. The transverse mode profile of cladding modes spreads to the cladding of the fiber and interacts with the outer medium interface. Therefore, cladding mode resonance frequencies depend on the refractive index of the outer medium in addition to other parameters. This feature can be exploited for refractive sensing applications [7]. In our device, the external coating is a layer of SWNTs, and interaction with light is allowed through cladding mode resonances. As SWNTs are a nonlinear material for optical wavelengths, the outer refractive index depends on the light intensity and an intensity-dependent TFBG transmission response is obtained. Figure $\underline{1(b)}$ depicts the SWNT-coated optical fiber structure.

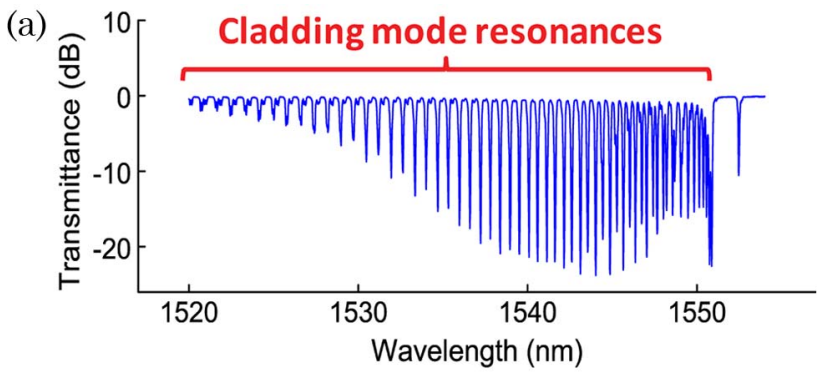

(b)

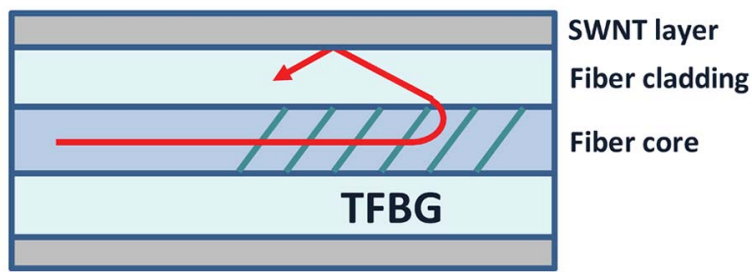

Fig. 1. (Color online) (a) Measured linear transmission response of a $4 \mathrm{deg}$ tilted fiber Bragg grating. (b) Nanotubecoated TFBG structure. Light for which the wavelength matches a cladding mode resonance couples to a cladding mode, interacting with the outer SWNT layer. 
Deposition of the SWNT coating was done either by dip-coating in a suspension of SWNTs in $N, N$ dimethylformamide (DMF) (dip-coated), or by collecting sections of a freely floating SWNT film around the optical fiber cladding (film-wrapped). Both approaches used high purity SWNTs $(\sim 1.3 \mathrm{~nm}$ in diameter and $>1 \mu \mathrm{m}$ in length) produced by a laser-oven method [8] and deposited onto single-mode fibers with a TFBG written in them. Both gratings had a length of $20 \mathrm{~mm}$, a tilt angle of $4 \mathrm{deg}$ and a center wavelength of $1552 \mathrm{~nm}$. They were fabricated using a phase mask and a $248 \mathrm{~nm}$ pulsed irradiation from a krypton fluoride excimer laser. An example of TFBG transmittance in this study, measured when injecting light from a $1550 \mathrm{~nm}$ centered broadband source into an uncoated fiber, is shown in Fig. 1(a).

For dip-coating, $\sim 10 \mathrm{mg}$ SWNTs were first functionalized by bath sonication $(2 \mathrm{~h})$ in $70 \%$ nitric acid. These SWNTs were recovered by filtration, rinsed with nanopure water $(>18 \Omega M \mathrm{~cm})$ and by bath sonication $(2 \mathrm{~h})$ in nanopure water, and then redispersed by sonication in $10 \mathrm{~mL}$ DMF. After the acid treatment, the SWNTs dispersed readily in DMF producing a black suspension. The optical fibers were then coated by dipping the section containing the TFBG, sequentially, in a $0.1 \mathrm{wt} . \%$ aqueous solution of 3-aminopropyltriethoxysilane (APTES), followed by the SWNT/DMF suspension, and finally in pure water. APTES has been used in the preparation of carbon nanotube networks on glass, silicon, and polyethylene terephthalate. In that approach, submerging the substrate in an ATPES solution results in the formation of an amino-terminated, silanized surface. This surface is positively charged and the carboxylic-functionalized SWNTs adsorb readily. The last step of water immersion was intended to homogenize the SWNT distribution and provide a well-known external medium when measuring TFBG transmission spectra. Repeated dipping cycles were used to increase the amount of SWNT coating and the deposition was monitored by measuring the transmission spectrum of the grating after each of ten dipping cycles. Cladding mode resonances are related to the outer medium properties; therefore, the am-

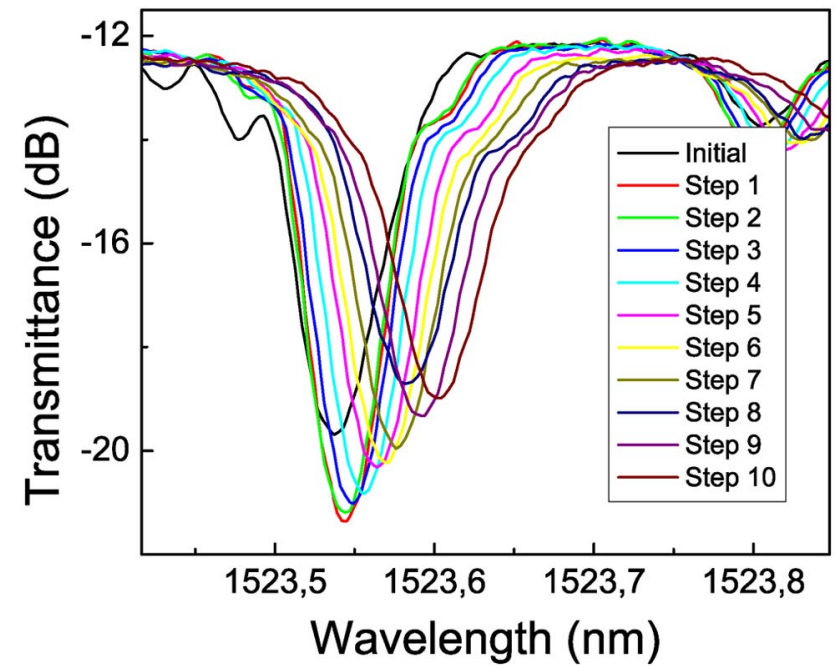

Fig. 2. (Color online) Cladding mode resonance monitored for one to ten dip-coating cycles. All responses are wavelength and amplitude equalized with regard to the Bragg resonance. ount of deposited SWNTs will be reflected in those resonances.

Figure 2 shows, in detail, one high-order cladding mode centered at $1523.6 \mathrm{~nm}$ for each dipping step. To quantitatively compare resonance wavelength shifts and depth changes, all spectra have been normalized in amplitude and wavelength to the Bragg mode resonance (which is inherently immune to coating and external media changes). A clear shift ( $\sim 67 \mathrm{pm})$ of the cladding mode resonance towards higher wavelength is observed. Monitoring this relative shift of cladding mode resonance wavelength provides a direct indicator of SWNT coating thickness for consecutive dip-coating cycles. The amplitude of the resonance depends on many factors but mostly on the overlap between the core mode, cladding mode, and index modulation. When the first carbon nanotubes are deposited, the cladding mode field amplitude is more strongly perturbed because of the new boundary condition. This justifies the amplitude increase observed between the initial state and step 1 in Fig. 2, whereas subsequent dipping steps decrease the depth.

For the film-wrapped TFBG, a floating SWNT film was first prepared as previously described [9]. In this case SWNTs $(4 \mathrm{mg} / \mathrm{l})$ were dispersed in a $2 \mathrm{wt} . \%$ aqueous sodium cholate solution, followed by production of a thin film by vacuum filtration of $\sim 10 \mathrm{ml}$ through a cellulose acetate filter membrane $(47 \mathrm{~mm}$ diameter, $0.22 \mu \mathrm{m}$ pore size), and then the film was detached by being submersed in water. Part of the floating film was collected by slowly lifting and rotating the optical fiber as it was pulled through the film in order to obtain a SWNT coating over the TFBG. The film tore as it was lifted away from the water surface, but the method still allowed for efficient collection of a much thicker coating, which is visibly dark gray. Because of the manual procedure of this method, the dip-coating approach is expected to provide better reproducibility.

Both SWNT coatings were examined by scanning electron microscopy (SEM) imaging and Raman spectroscopy, and the results are shown in Fig. 3, proving the
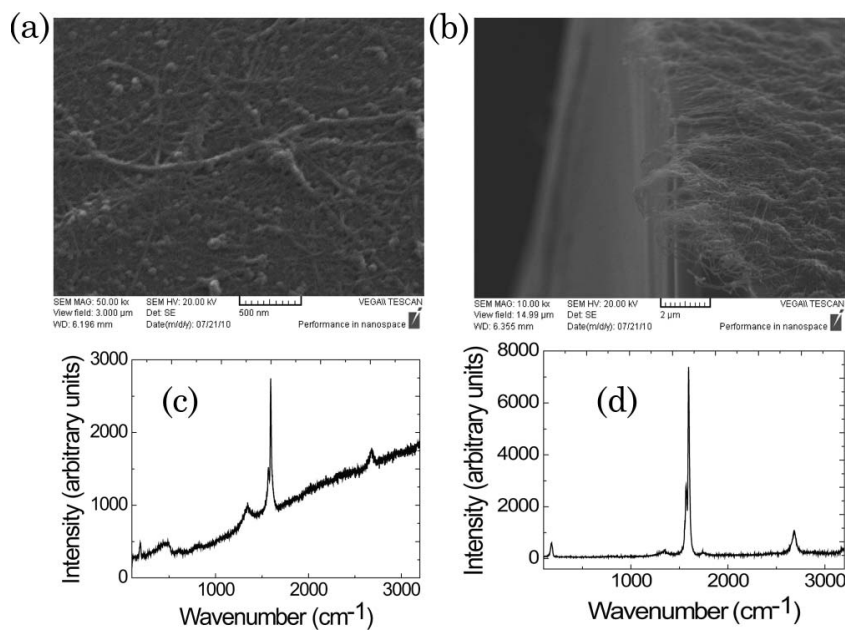

Fig. 3. SEM images of deposited nanotubes on the optical fiber cladding for (a) dip-coating and (b) wrapping techniques. In (b) the edge of a cleaved fiber is depicted. Raman spectroscopy for (c) dip-coating and (d) wrapping techniques. The background slope is due to the relatively small number of SWNTs in the sampling volume that is achieved with the sparse coating method. 


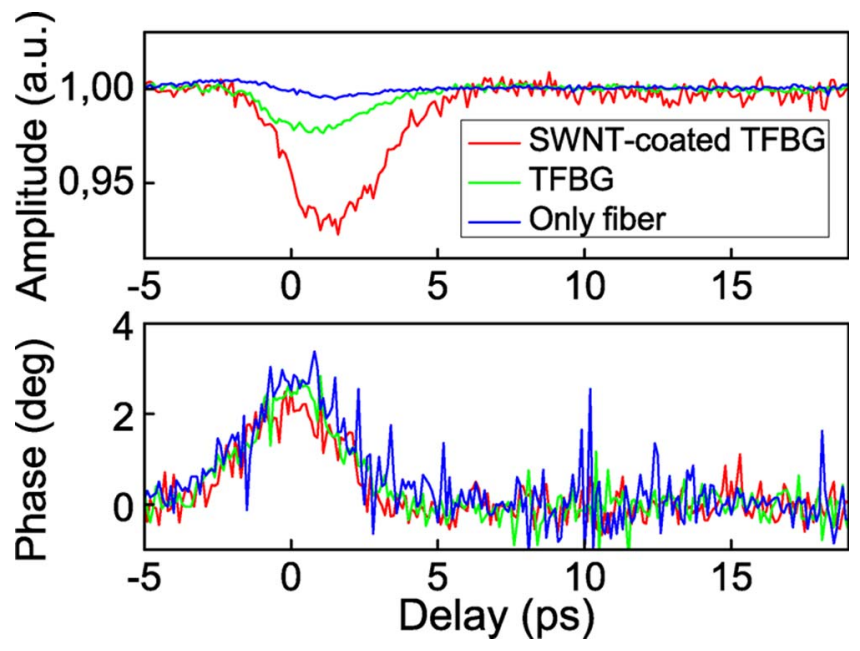

Fig. 4. (Color online) Nonlinear amplitude and phase responses of the SWNT-coated TFBG, of an uncoated regular 4 deg TFBG, and of a standard fiber.

presence of nanotubes on the fiber surface. From SEM images and variation of the intensity of the Raman spectra from location to location, we can deduce that dipcoating provides a thinner and more homogeneous layer of SWNTs on the fiber. In contrast, the wrapping technique produces a much thicker coating, but with less control of the uniformity of the wrapping.

An experiment was performed to demonstrate the interaction of light with the SWNT coating via TFBG cladding mode resonances. Nonlinear effects were measured with a pump-probe phase-sensitive setup described elsewhere [10]. In this technique, a $1 \mathrm{ps}$ laser pulse is divided into three pulses, one of them intense (pump) and two identical weak ones (a reference and a probe). All three pulses are combined by setting the pump very close in time to the probe, making this delay variable with a delay line. The amplitude and phase of the probe with respect to the reference pulse is measured with a heterodyne technique and monitored as a function of the delay of the pump. The pulsed light injected into the TFBG had $-3.5 \mathrm{dBm}$ and $1535 \mathrm{~nm}$ of average power and wavelength respectively, providing $22.5 \mathrm{~W}$ of peak power in the fiber. The $1 \mathrm{ps}$ duration determines the measurement resolution. The optical fiber coated by the wrapping method was measured since it provides a thicker coating compared to dip-coating and, therefore, larger nonlinear effects are expected.

Figure $\underline{4}$ shows the nonlinear responses of the SWNT film-wrapped TFBG, a regular TFBG without coating, and standard fiber. These results demonstrate that interaction of the pump with the nonlinear cladding, as mediated by the TFBG, is sufficient to modulate the amplitude of the probe by more than $6 \%$ (12\% in intensity) on a picosecond time scale. The phase response remains constant, since it is mainly due to the Kerr effect from the fiber silica. Therefore, linear and nonlinear transmission responses of the tilted grating induce no noticeable effect in the phase of the transmitted pulse. We also have checked that the magnitude of the amplitude effect depends on the relative spectra of the TFBG and of the probe light, indicating possible optimization strategies.

In conclusion, SWNT-coated single-mode optical fibers have been proposed using, for the first time to our knowledge, a standard optical fiber with a TFBG for nonlinear applications. Two simple techniques for nanotube deposition on the cladding surface have been carried out. The use of tilted fiber Bragg grating assisted the interaction of transmitted light with the SWNT-coating. The measurement of the cladding mode resonances allowed us to monitor the progressive deposition of nanotubes on the cladding for a dip-coating method. The wrapping method provided a simple and innovative way to deposit thick SWNT layers on optical fibers. Finally, picosecond nonlinear effects were measured by means of pumpprobe measurements, showing an effective intensity modulation via TFBG cladding mode coupling.

This work was financially supported by the European Commission under the FP7 EURO-FOS Network of Excellence (ICT-2007-2-224402), the Ministerio de Educación y Ciencia SINADEC project (TEC2008-06333), and the Natural Sciences and Engineering Research Council of Canada (NSERC). The work of G. E. Villanueva was supported by the Ministerio de Educación y Ciencia Formación de Profesorado Universitario programs. The work of P. Pérez-Millán was supported by the Juan de la Cierva program, JCI-2009-05805.

\section{References}

1. Y. Sakakibara, A. Rozhin, H. Kataura, Y. Achiba, and M. Tokumoto, Jpn. J. Appl. Phys. 44, 1621 (2005).

2. K. K. Chow, S. Yamashita, and Y. W. Song, Opt. Express 17, 7664 (2009).

3. S. Set, H. Yaguchi, Y. Tanaka, and M. Jablonski, IEEE J. Sel. Top. Quantum Electron. 10, 137 (2004).

4. K. K. Chow, M. Tsuji, and S. Yamashita, Appl. Phys. Lett. 96, 061104 (2010).

5. K. K. Chow and S. Yamashita, Opt. Express 17, 15608 (2009).

6. S. Y. Choi and F. Rotermund, H. Jung, K. Oh, and D. Yeom, Opt. Express 17, 21788 (2009).

7. C. Chan, C. Chen, A. Jafari, A. Laronche, D. J. Thomson, and J. Albert, Appl. Opt. 46, 1142 (2007).

8. C. Kingston, Z. Jakubek, S. Dénommée, and B. Simard, Carbon 42, 1657 (2004).

9. M. Jakubinek, M. Johnson, M. White, J. Guan, and B. Simard, J. Nanosci. Nanotechnol. 10, 8151 (2010).

10. T. Vallaitis, C. Koos, R. Bonk, W. Freude, M. Laemmlin, C. Meuer, D. Bimberg, and J. Leuthold, Opt. Express 16, 170 (2008). 